ARTICLE

https://doi.org/10.1038/s41467-019-09020-4

\title{
A widely distributed diheme enzyme from Burkholderia that displays an atypically stable bis-Fe(IV) state
}

Kimberly Rizzolo ${ }^{1}$, Steven E. Cohen (10 ${ }^{2}$, Andrew C. Weitz ${ }^{3}$, Madeline M. López Muñoz ${ }^{1}$, Michael P. Hendrich ${ }^{3}$, Catherine L. Drennan (iD) ${ }^{2,4,5} \&$ Sean J. Elliott (i) ${ }^{1}$

Bacterial diheme peroxidases represent a diverse enzyme family with functions that range from hydrogen peroxide $\left(\mathrm{H}_{2} \mathrm{O}_{2}\right)$ reduction to post-translational modifications. By implementing a sequence similarity network (SSN) of the bCCP_MauG superfamily, we present the discovery of a unique diheme peroxidase BthA conserved in all Burkholderia. Using a combination of magnetic resonance, near-IR and Mössbauer spectroscopies and electrochemical methods, we report that BthA is capable of generating a bis-Fe(IV) species previously thought to be a unique feature of the diheme enzyme MauG. However, BthA is not MauG-like in that it catalytically converts $\mathrm{H}_{2} \mathrm{O}_{2}$ to water, and a $1.54-\AA$ resolution crystal structure reveals striking differences between BthA and other superfamily members, including the essential residues for both bis-Fe(IV) formation and $\mathrm{H}_{2} \mathrm{O}_{2}$ turnover. Taken together, we find that BthA represents a previously undiscovered class of diheme enzymes, one that stabilizes a bis-Fe(IV) state and catalyzes $\mathrm{H}_{2} \mathrm{O}_{2}$ turnover in a mechanistically distinct manner.

\footnotetext{
${ }^{1}$ Boston University, Department of Chemistry, Boston, MA 02215, USA. ${ }^{2}$ Massachusetts Institute of Technology, Department of Chemistry, Cambridge, MA 02139, USA. ${ }^{3}$ Carnegie Mellon University, Department of Chemistry, Pittsburgh, PA 15213, USA. ${ }^{4}$ Massachusetts Institute of Technology, Department of Biology, Cambridge, MA 02139, USA. ${ }^{5}$ Howard Hughes Medical Institute, Cambridge, MA 02139, USA. Correspondence and requests for materials should be addressed to S.J.E. (email: elliott@bu.edu)
} 
$\mathrm{M}$ icroorganisms utilize a vast array of metal-containing proteins to protect the cell from reactive oxygen species (ROS). Proteins containing heme cofactors are well known for incorporating hydrogen peroxide $\left(\mathrm{H}_{2} \mathrm{O}_{2}\right)$ in catalytic processes as a means to remove the xenotoxic oxidant generated via the incomplete reduction of dioxygen $\left(\mathrm{O}_{2}\right)^{1}$. Within Gramnegative bacteria, cell viability is maintained in part by cytochrome $c$ peroxidases (bCCPs), which convert $\mathrm{H}_{2} \mathrm{O}_{2}$ to $\mathrm{H}_{2} \mathrm{O}$ in the periplasm, taking electrons from small electron transfer proteins ${ }^{2}$. bCCPs are structurally and mechanistically distinct from well-studied monoheme plant and fungal peroxidases in that they house two $c$-type heme prosthetic groups covalently bound to the protein backbone by a CXXCH-binding motif ${ }^{3,4}$. However, the bCCP superfamily also includes MauG, an enzyme that shares the same canonical bCCP fold and many similarities in key amino acid positions, yet catalyzes strikingly different chemistry: where bCCPs only appear to be capable of oxidizing a redox partner protein of relatively low redox potential $(\sim 250 \mathrm{mV})$, MauG is responsible for the oxidation of specific Trp residues $(\sim 1 \mathrm{~V})$ to form the catalytic cofactor tryptophan tryptophylquinone (TTQ) in the precursor protein of methylamine dehydrogenase (preMADH $)^{5-7}$. A molecular perspective of how bCCPs and MauG differ in terms of the functionality as peroxidases has been long attributed to differences in the coordination environment of the coordinately saturated (6c) heme. Here, we further demonstrate that those differences are not the sole determiners of reactivity, and provide evidence of a class of diheme enzymes that share properties with classic bCCPs and MauGs, but is also distinct structurally and mechanistically.

In both MauG and bCCPs, the peroxidatic active site is a fivecoordinate (5c), low potential (LP) heme, which serves as the site of $\mathrm{H}_{2} \mathrm{O}_{2}$ binding (Fig. 1a). However, the six-coordinate (6c), high potential (HP) heme site that assists in electron transfer (ET) to and from the active site differs with respect to the ligation environment. In bCCPs, such as the well-characterized enzyme from Nitrosomonas europaea $(\mathrm{Ne})^{8}$, the (6c) heme is Met-His ligated, whereas in MauG, it is Tyr-His ligated ${ }^{7}$. These variations play out in terms of critical differences in chemistry, which correlates with the redox potentials observed when reducing equivalents are introduced into the protein system. In canonical bCCPs, the presence of the Met-His ligated HP heme means that the enzyme can be prepared in a very stable $\mathrm{Fe}_{\mathrm{HP}}{ }^{\mathrm{II}} \mathrm{Fe}_{\mathrm{LP}}{ }^{\mathrm{III}}$ oxidation state, which contrasts with MauG. Instead, the two hemes of MauG are close in redox potential ${ }^{6}$ and are termed $5 c$ and $6 \mathrm{c}$ accordingly; the $\mathrm{Fe}_{5 \mathrm{c}}{ }_{\mathrm{CI}}^{\mathrm{II}} \mathrm{Fe}_{6 \mathrm{c}}{ }_{\mathrm{III}}$ form of the enzyme is not stable, which appears to be critical for MauG function.

MauG's function is the six-electron oxidation of two tryptophan residues ( $\beta \operatorname{Trp} 57$ and $\beta \operatorname{Trp} 107)$ in preMADH, achieved by long-range ET through the two heme sites of MauG, a precise and long-distance chemistry that earned MauG the moniker 'Nature's sniper' (Fig. 1b) ${ }^{9-11}$. Studies of MauG have focused on the characterization of a bis-Fe(IV) species, formulated as $\mathrm{Fe}^{\mathrm{IV}} \mathrm{Fe}^{\mathrm{IV}}$ $=\mathrm{O}$, which is formed upon reaction with $\mathrm{H}_{2} \mathrm{O}_{2}$ in the absence of substrate, preMADH ${ }^{12}$. The bis-Fe(IV) state is a unique alternative to the extensively studied compound I (Cpd I) of monoheme cytochrome P450 enzymes and monoheme plant peroxidases ${ }^{13,14}$. Currently, the proposed mechanism of MauG involves $\mathrm{H}_{2} \mathrm{O}_{2}$ binding to the $5 \mathrm{c}$ heme to generate an unstable Cpd I species, which is immediately reduced to Cpd II via oxidation of the $6 \mathrm{c}$ heme to $\mathrm{Fe}(\mathrm{IV})$, while still maintaining Tyr-His ligation ${ }^{12}$. The formation of the bis-Fe(IV) state has set MauG apart from the canonical bCCPs and, more excitingly, highlights the diverse chemistry present in microorganisms.

We have taken a bioinformatics approach to aid a comparison of MauG structure and function with respect to canonical bCCPs, and in doing so, we have identified family members of unusual reactivity. MauG is encoded by one of 11 genes present in the mau cluster ${ }^{15}$, and in looking for close relatives of MauG, (Fig. 1c), we recognized an uncharted region of potential $\mathrm{H}_{2} \mathrm{O}_{2}$ reactivity, obligately found in soil microbes of the genus
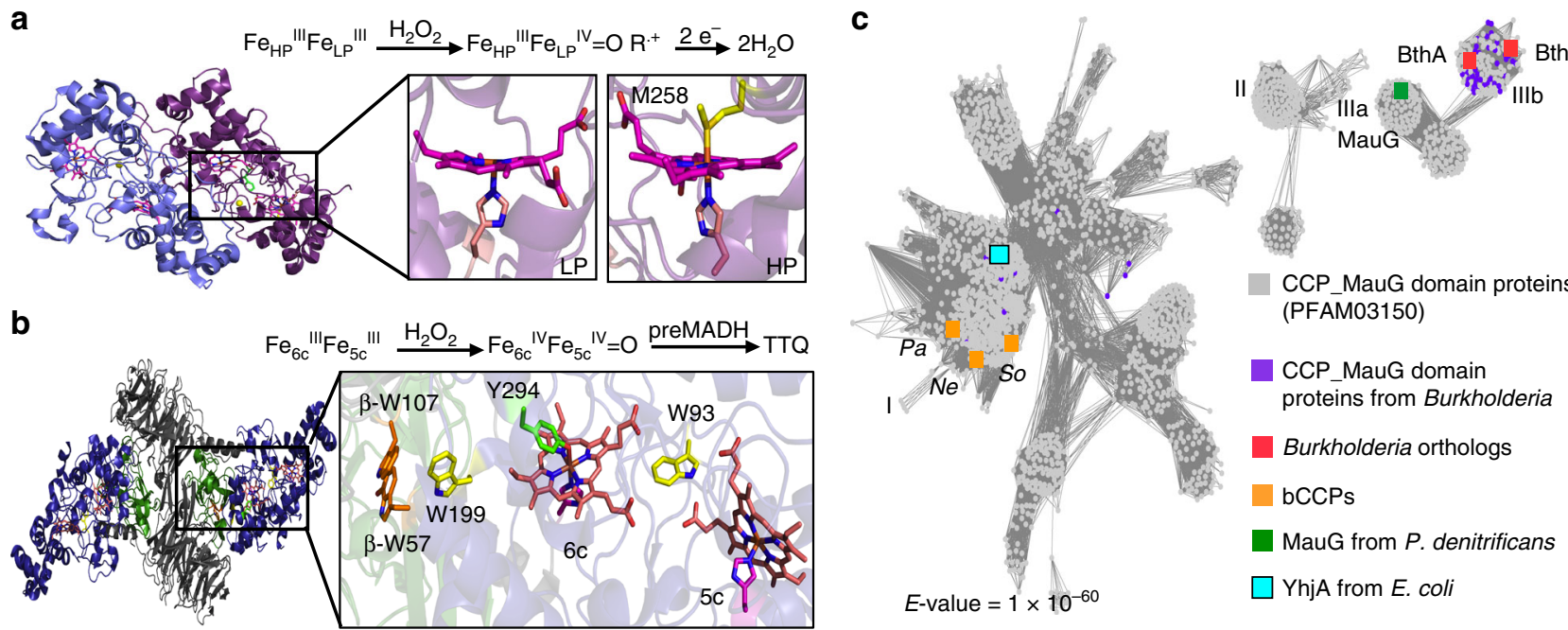

Fig. 1 SSN of CCP_MauG domain containing proteins. a Active site domain of bCCPs. Reaction scheme indicates oxidation states achieved for reaction of bCCPs with $\mathrm{H}_{2} \mathrm{O}_{2}$. Upon addition of $\mathrm{H}_{2} \mathrm{O}_{2}$, a compound I intermediate is formed, where an electron is lost from a nearby tryptophan as is suggested for Ne. Crystal structure of Ne peroxidase (PDB: 1IQC) is shown as dimer (purple). Inset shows structure of the six-coordinate, high potential (HP) heme (right) and five-coordinate, low potential (LP) heme (left). $\mathbf{b}$ Active site domain of MauG. Reaction scheme shows overall oxidation state achieved for reaction of MauG with $\mathrm{H}_{2} \mathrm{O}_{2}$ in the presence of preMADH. Crystal structure of overall fold of MauG is shown in complex with preMADH (PDB: $3 L 4 M$ ). Inset shows diheme active site of MauG, including key tryptophan residues that are post-translationally modified in preMADH ( $\beta$-W107, $\beta$-W57) after reaction with $\mathrm{H}_{2} \mathrm{O}_{2}$. Six-coordinate heme (6c) is shown with Tyr-His ligation. c SSN generated for CCP_MauG containing proteins with $E$-value $=1.0 \times 10^{-60}$. Graphical representation of network created using Cytoscape. Main protein clusters, where each node represents and individual sequence, are identified by roman numerals. Cluster I includes canonical bCCPs, Cluster IIla includes MauG from P. denitrificans and other known orthologs, and Cluster IIIb includes diheme enzymes (BthA and BthB from B. thailandensis are indicated) conserved in Burkholderia 
Burkholderia, an opportunistic bacterium, with known implications in serious diseases such as cystic fibrosis ${ }^{16}$. Herein we report the characterization of the class A diheme enzyme BthA (Uniprot ID: Q2T6B0) from B. thailandensis. Our efforts reveal that BthA is a peroxidase, capable of both $\mathrm{H}_{2} \mathrm{O}_{2}$ turnover when in the presence of an external electron source, yet also generates the bis-Fe(IV) species, as determined by Near-infrared (NIR) and Mössbauer spectroscopy. Despite the similarities between BthA and MauG reactivity shown below, we find that the genomic context of the bthA gene reveals it is not encoded within a mau operon. While bthA is highly conserved in all strains of Burkholderia it is always found downstream of a putative phosphatase partner of unknown function (Supplementary Fig. 1), but with annotation that indicates extra-cellular localization. bth $\mathrm{A}$ is predicted to be localized in the periplasm, as it contains a canonical leader sequence associated with the periplasmic maturation of $c$-type cytochromes in Gram-negative microorganisms. Intriguingly, a $1.54-\AA$ resolution crystal structure presented herein confirmed Tyr-His ligation of heme $6 c$, yet surprisingly, revealed lack of a clear ET network of residues between the two heme sites, assumed to be essential for generation of a bis-Fe(IV) state. Unlike the canonical tryptophan residue present midway between the heme centers of both bCCPs and MauG, the BthA structure provides evidence for a serine residue in this highly conserved position, which was shown not to inhibit the bis-Fe(IV) formation or peroxidase activity when mutated to alanine. Taking a biochemical and biophysical approach to exploring uncharacterized members of the peroxidase superfamily, we introduce a diheme enzyme to the peroxidase superfamily involved in mechanistically distinct oxidation chemistry.

\section{Results}

A sequence similarity network of the CCP_MauG superfamily. A SSN of the bacterial peroxidase superfamily was generated by use of the enzyme function initiative (EFI) sequence similarity tool ${ }^{17,18}$. Our network comprises protein sequences found with PFAM domain CCP_MauG, identified with the code PF03150. Pooling over 6000 protein sequences, $E$-values were lowered in steps of $10^{-10}$ to arrive at a stringent $E$-value cutoff value of $10^{-60}$, which results in a representative isofunctional network possessing several protein-sequence clusters, where each node represents a set of proteins with $80 \%$ or higher sequence identity (Fig. 1c). Using Cytoscape ${ }^{19}$ to represent the network graphically, canonical bCCPs were mapped to Cluster I, which includes the structurally characterized enzymes from Ne, Pseudomonas aeruginosa $(\mathrm{Pa})$, and Shewanella oneidensis (So), despite known differences in redox-based activation for these bCCP members ${ }^{8,20,21}$. The main cluster also contains triheme peroxidase YhjA from $E$. coli, which has recently been reported to use $\mathrm{H}_{2} \mathrm{O}_{2}$ as a respiratory substrate under anoxic conditions ${ }^{22}$. The map of proteins conserved in the different clusters of the network suggests a difference in protein function as the primary sequence of the peroxidases evolve. As suspected, MauG from $P$. denitrificans is located in a separate cluster from the canonical bCCPs (Cluster IIIa, Fig. 1c). True MauG orthologs, where genome analysis provides evidence for a mau operon, are largely found in Cluster IIIa. Surprisingly, these MauG orthologs are also connected to Cluster IIIb, which represents sequences that share $~ 30 \%$ identity to MauG and $\sim 25 \%$ identity to the canonical bCCPs of Cluster I (See Supplementary Table 1). These sequences contain two $\mathrm{CXXCH}$ motifs, are found obligately in the genus Burkholderia, and further analysis of Cluster IIIb identified two separate diheme proteins present in all Burkholderia strains. Exploration of the Burkholderia genomes revealed lack of a conserved mau operon, suggesting an alternate chemical role for these diheme bCCPs.
We term these two related Burkholderia proteins as Class A (found on chromosome II), and Class B (chromosome I). A sequence alignment of the $\mathrm{A}$ and $\mathrm{B}$ enzyme from $\mathrm{B}$. thailandensis shows a $40 \%$ sequence identity between the proteins. Both BthA and $\mathrm{BthB}$ possess the conserved tyrosine residue predicted to ligate the $6 \mathrm{c}$ heme site, and yet have clear differences with MauG or other bCCP family members: their N-termini are rich in alanine residues, suggesting hydrophobicity (though signal prediction servers suggest both are exported periplasmically), and both enzymes possess conserved cysteine residues near their C-termini (and the structure presented below reveals an unforeseen disulfide linkage). These traits may yield clues to better understand the $\mathrm{H}_{2} \mathrm{O}_{2}$ chemistry observed for BthA.

BthA as a redox mimic of MauG. Establishing the optical and electrochemical properties of BthA is important for establishing how this diheme enzyme relates to known bacterial peroxidases. BthA purifies with both heme irons in the oxidized state (diferric), with a Soret maximum at $403 \mathrm{~nm}$, and a broad feature centered at $630 \mathrm{~nm}$ believed to signify a high-spin, five-coordinate heme site. Additionally, BthA has four distinct peaks at 495, 538, 570 , and $610 \mathrm{~nm}$, which we attribute to Tyr-His ligation for sixcoordinate ferric heme centers based on similar literature values reported for the heme-binding protein $\mathrm{Has}^{23}$. A reductive titration with up to $20 \mathrm{mM}$ ascorbate added to the diferric state results in no spectral changes, highlighting an inability of BthA to achieve the optically characterized semi-reduced $\left(\mathrm{Fe}_{\mathrm{HP}}{ }^{\mathrm{II}} \mathrm{Fe}_{\mathrm{LP}} \mathrm{III}\right)$ state observed for canonical bCCPs achieved when treated with only $1 \mathrm{mM}$ sodium ascorbate (Fig. 2a). Upon reduction with dithionite, the electronic absorption spectrum of BthA is atypical of canonical bCCPs and MauG, particularly with respect to the $\alpha / \beta$ band region (Fig. 2b). Reduction of BthA results in a Soret shift from 403 to $428 \mathrm{~nm}$, and generates a broad feature at $550 \mathrm{~nm}$, as opposed to sharp $\alpha / \beta$ bands at 550 and $524 \mathrm{~nm}$, respectively, reported for most reduced cytochrome $c$ proteins ${ }^{24}$. Dithionite-reduced BthA was shown to bind the exogenous ligand carbon monoxide (CO), demonstrated by (1) a blue shift in the Soret to $416 \mathrm{~nm}$; (2) increase in extinction coefficient; and (3) formation of a broadened band in the $\alpha / \beta$ band region at $550 \mathrm{~nm}$. Collectively, these data suggest a closer relationship of BthA to MauG than any known bCCP; a salient finding here is the inability of ascorbate to reductively generate a $\mathrm{Fe}_{\mathrm{HP}}{ }^{\mathrm{II}} \mathrm{Fe}_{\mathrm{LP}} \mathrm{III}$ state with a diagnostic Soret shoulder at $414 \mathrm{~nm}$ and the partial appearance of $\alpha / \beta$ bands ${ }^{8}$.

The electrochemical properties of the heme centers of BthA were determined in order to correlate the reduction potentials to the spectroscopic features observed for diferric and diferrous forms of the protein. Both direct electrochemistry and coupled optical titrations revealed two redox couples that are close in potential, such that producing a single semi-reduced state (i.e., like bCCP) is unlikely if not impossible. By use of protein film voltammetry (Fig. 2c), the redox characteristics of the two heme centers can be fit to a model of two species with potentials of -121 and $-165 \mathrm{mV}$ vs. SHE, consistent with (though not identical to) what has been observed for MauG, when the protein is reduced with transitions occurring at -159 and $-244 \mathrm{mV}$ vs. $\mathrm{SHE}^{6}$. Parallel spectroelectrochemical titrations utilizing an optically transparent thin-layer electrode (OTTLE) ${ }^{25}$ yielded similar BthA redox transitions of -100 and $-220 \mathrm{mV}$ (Supplementary Fig. 2).

Verification of MauG-like heme environments in the resting state. As indicated by the absorption spectrum for as-isolated protein, BthA purifies in the diferric state and both heme centers were found to be active by electron paramagnetic resonance 

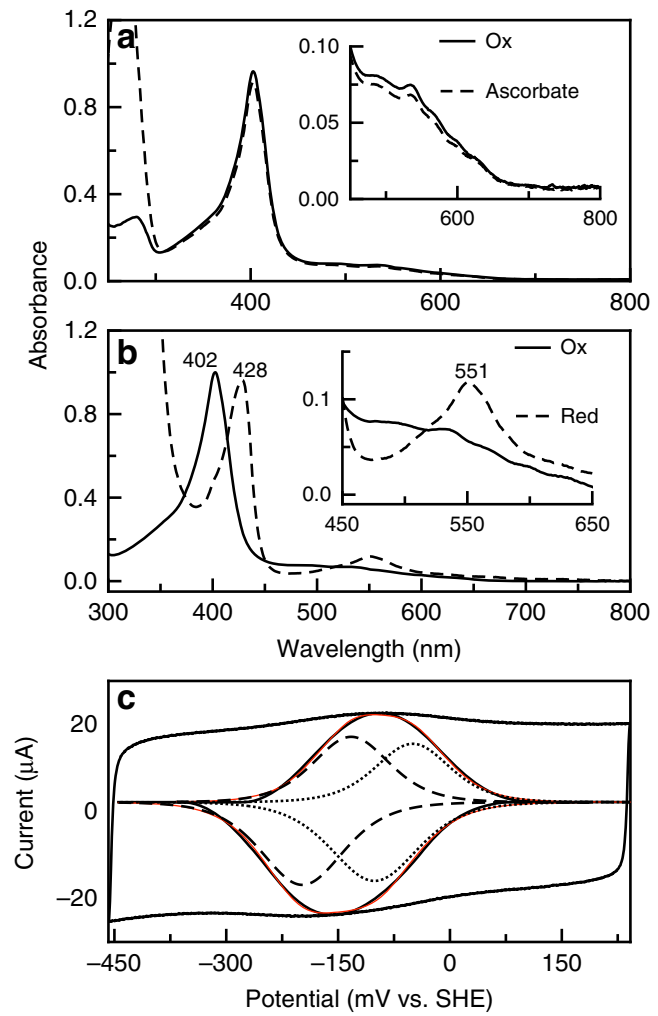

Fig. 2 Optical characterization of BthA. a Oxidized BthA (solid line) and ascorbate treated (dashed line). Ascorbate was added to $20 \mathrm{mM}$ final concentration before scan was collected. Inset shows range from 450 to $800 \mathrm{~nm}$, indicating no signs of reduction to ferrous form. b Oxidized BthA (solid line) and dithionite treated (dotted line). Dithionite was added to a final concentration of $1 \mathrm{mM}$. Inset shows peak formed at $550 \mathrm{~nm}$. c Deconvoluted spectra of BthA on PGE. Voltammogram of BthA on

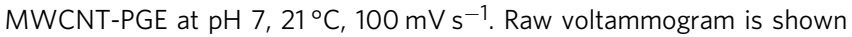
(black) with the baseline subtracted (black, inset) with fit for the overall signal (red) and individual species (dotted and dashed line)

(EPR) spectroscopy (Fig. 3a). The X-band EPR spectrum of BthA is similar to MauG, displaying two distinct heme centers as a mixture of high- and low-spin species. Quantitative simulations of the EPR spectra are overlaid on the experimental spectrum (Fig. 3a). The high-spin (HS) species gave $g$-values near $g=6$ and 2 , indicative of an axial $(E / D<0.001) 5$-coordinate $(5 c)$ heme, accounting for $45 \%$ of the $\mathrm{Fe}(\mathrm{III})$ in the sample. The low-spin (LS) species had $g$-values of $2.54,2.19,1.86$, accounting for $55 \%$ of the $\mathrm{Fe}$ (III) in the sample. The total heme concentration was in agreement with the protein concentration. The observed ratio of the two species can be dependent on the method of purification, wherein some of the $5 \mathrm{c}$ site heme converts to $6 \mathrm{c}$ as a function of $\mathrm{pH}$.

The Mössbauer spectrum of diferric BthA in a field of $45 \mathrm{mT}$ recorded at $4 \mathrm{~K}$ is shown in Fig. $3 \mathrm{~b}$. The spectrum showed a mixture of $S=5 / 2$ and $S=1 / 2 \mathrm{Fe}$ (III) species in approximately the same ratio as observed from the EPR spectrum. However, without accompanying high-field data, a unique fit for the HS and LS Fe(III) hemes could not be obtained ${ }^{26}$. At higher temperature $(100 \mathrm{~K})$, the electronic relaxation was intermediate relative to the nuclear precession frequency and gave a broad featureless spectrum.

BthA produces a bis-Fe(IV) species like MauG. To date, MauG is the only reported diheme enzyme capable of forming a bis-Fe
(IV) state when reacted with $\mathrm{H}_{2} \mathrm{O}_{2}{ }^{12}$. Optically, generation of this state can be monitored via formation of a peak at $950 \mathrm{~nm}$, defined by a charge resonance stabilization between the $5 c$ and $6 c$ hemes due to the reversible oxidation and reduction of Trp93 located midway between the hemes ${ }^{27}$. This NIR feature occurs concomitant with a shift in Soret and growth of charge transfer (CT) bands. The lifetime of the bis-Fe(IV) species in MauG was 10 min, and the reduction back to the diferric state was achieved by oxidative damage of nearby Met residues associated with the $5 \mathrm{c}$ heme $\mathrm{e}^{28}$. BthA is also capable of generating the putative bis-Fe(IV) species and produces an electronic absorption spectrum similar to peroxide-treated MauG (Fig. 3d). Upon treatment of BthA with $\mathrm{H}_{2} \mathrm{O}_{2}$ a peak at $960 \mathrm{~nm}$ immediately appeared coupled with a decrease in Soret intensity and shift from 403 to $411 \mathrm{~nm}$ in addition to the appearance of CT bands at 524 and $560 \mathrm{~nm}$. However, we found two striking differences between this newly generated species of BthA when compared to MauG. First, the rate for enzyme relaxation back to the diferric state (as monitored by the Soret position and intensity) is markedly slower than MauG. Monitoring the decay of the $960 \mathrm{~nm}$ feature and return of Soret to $403 \mathrm{~nm}$, this oxidized state can persist for over one hour (Fig. 3e). The decay of the $960 \mathrm{~nm}$ peak was fit to a single exponential rate constant of $0.014 \mathrm{~min}^{-1}$, whereas the $403 \mathrm{~nm}$ peak was fit to a single exponential rise with a rate constant of $0.04 \mathrm{~min}^{-1}$. As discussed below, this observation challenges the convention of the proposed reactivity of a $\mathrm{Fe}^{\mathrm{IV}} \mathrm{Fe}^{\mathrm{IV}}=\mathrm{O}$ species as a hot oxidant.

The long-lived nature of the NIR feature at $960 \mathrm{~nm}$ prompted further investigation of this unusually stable bis-Fe(IV) state by EPR and Mössbauer spectroscopies. Addition of one equivalent $\mathrm{H}_{2} \mathrm{O}_{2}$ to an EPR sample of BthA resulted in a $50 \%$ loss of signal intensity for both the HS and LS features, suggestive of an oxidative event of each heme site to an EPR-silent Fe(IV) state. Samples of ${ }^{57} \mathrm{Fe}$-enriched BthA were treated with $\mathrm{H}_{2} \mathrm{O}_{2}$ and Mössbauer spectra were recorded at $4 \mathrm{~K}$ in a field of $45 \mathrm{mT}$. Upon exposure to one equivalent of $\mathrm{H}_{2} \mathrm{O}_{2}, 50 \%$ of the diferric signal was lost and replaced by two doublets having approximately equal areas and parameters: (1) $\delta=0.07 \mathrm{mM} \mathrm{s}^{-1}, \Delta \mathrm{Eq}=$ $1.70 \mathrm{mM} \mathrm{s}^{-1}$; and (2) $\delta=0.17 \mathrm{mM} \mathrm{s}^{-1}, \Delta \mathrm{Eq}=2.58 \mathrm{mM} \mathrm{s}^{-1}$. Addition of excess $\mathrm{H}_{2} \mathrm{O}_{2}$ (10 equiv.) resulted in complete conversion of the HS and LS species to the two new doublets (Fig. 3c). The parameters were indicative of the formation of $\mathrm{Fe}(\mathrm{IV})$ species and were similar to those of $\mathrm{MauG}^{12}$. Thus, in terms of optical and magnetic spectroscopies, a key similarity between BthA and MauG was established based on the presence of a bis-Fe(IV) species, which possesses kinetic stability over the course of minutes.

BthA as a potential peroxidase. Given the remarkable electronic similarity between BthA and MauG, the absence of a mau operon to support BthA's function and ubiquity in Burkholderia prompted us to investigate its potential enzymatic activity. MauG is considered a poor peroxidase ${ }^{5}$, and BthA shares only a $\sim 25 \%$ sequence identity with other canonical bCCP family members (e.g. enzymes from $\mathrm{Ne}$ or Geobacter sulfurreducens) ${ }^{29}$. Catalytic, steady-state reduction of $\mathrm{H}_{2} \mathrm{O}_{2}$ was assayed to address the putative role of $\mathrm{BthA}$ in $\mathrm{H}_{2} \mathrm{O}_{2}$ detoxification. Surprisingly, the kinetic parameters of $\mathrm{H}_{2} \mathrm{O}_{2}$ turnover indicate $\mathrm{BthA}$ is a very good peroxidase despite its spectroscopic and electrochemical parallels to MauG. For comparison to activity reported for bCCPs, activity was first determined with use of commercially available cytochrome $c$ from horse heart ( $\mathrm{HH}$ cyt $c$ ) (reduced) as the electron donor, but no trace of activity was detected. It is possible the molecular interaction between BthA and cyt $c$ is not favorable and, therefore, resulted in poor electron transfer. 

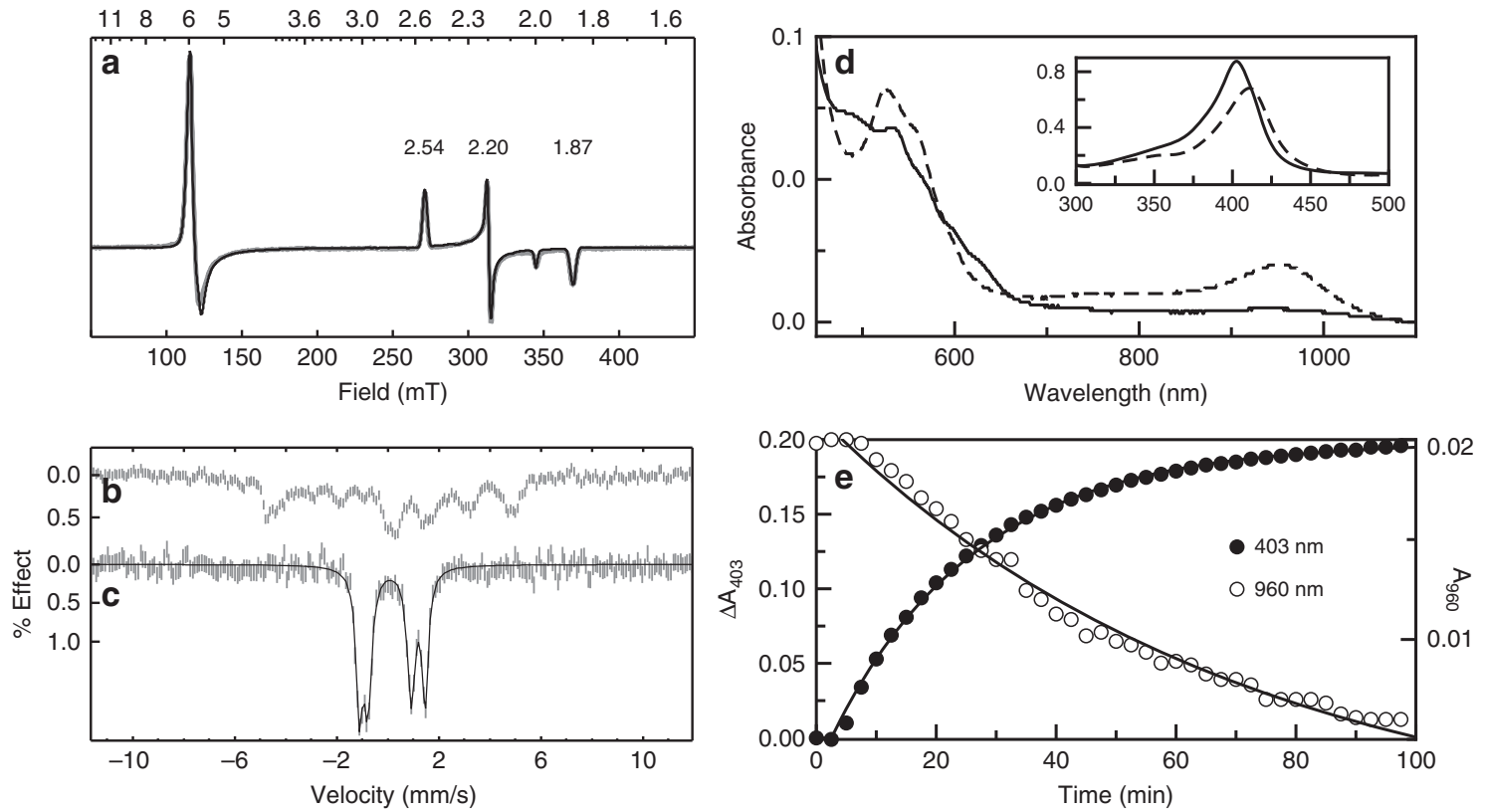

Fig. 3 Formation of a bis-Fe(IV) species in BthA. a EPR spectrum $(9.642 \mathrm{GHz}, 2 \mu \mathrm{W})$ of $0.8 \mathrm{mM}$ as-isolated Wt BthA recorded at $13 \mathrm{~K}$. See main text for simulation parameters and corresponding species concentrations. b Mössbauer spectrum and simulations of as-isolated ${ }^{57} \mathrm{Fe}$-enriched BthA recorded in an applied field of $45 \mathrm{mT}$ parallel to the incident $\gamma$-radiation, at a temperature of $4.2 \mathrm{~K}$. See main text for simulation parameters. c After addition of 10 equiv. $\mathrm{H}_{2} \mathrm{O}_{2}$ to the sample in $\mathbf{b}$. d NIR spectra of Oxidized BthA (solid line) and $\mathrm{H}_{2} \mathrm{O}_{2}$ treated (dashed line). After addition of $\mathrm{H}_{2} \mathrm{O}_{2}$, scans were collected every $2 \mathrm{~min}$ for $1.5 \mathrm{~h}$ (gray scans). The inset shows the shift in Soret from 403 to $411 \mathrm{~nm}$. Over time, the feature at $960 \mathrm{~nm}$ begins to decay, and the Soret shifts back to the $402 \mathrm{~nm}$ position, suggesting reduction back to the initial, diferric state. e Decay of the bis-Fe(IV) monitored by the change in absorbance for the 402 and $960 \mathrm{~nm}$ feature vs. time. The rise of the Soret back to the $403 \mathrm{~nm}$ (closed circles) and the $960 \mathrm{~nm}$ decay (open circles) are shown as a function of time. Data were fit to a single exponential rise/decay

Using ABTS as the artificial electron donor, activity was measurable. For BthA, $k_{\text {cat }} / K_{\mathrm{M}}=1.6 \times 10^{6} \mathrm{M}^{-1} \mathrm{~s}^{-1}$ with a relatively low $K_{\mathrm{M}}$ of $3.4 \pm 0.4 \mu \mathrm{M}$. These values are comparable to canonical bCCPs with $k_{\text {cat }} / K_{\mathrm{M}}$ values that range from $10^{6}-10^{8} \mathrm{M}^{-1} \mathrm{~s}^{-1}$. To validate this assay, we mutated a conserved residue near the $5 \mathrm{c}$ heme (Glu277 in BthA) that is known to act as an acid-base catalyst during peroxide turnover ${ }^{30}$. We found that the E277Q variant was inactive compared to the $\mathrm{Wt}$ enzyme (Supplementary Table 2), providing further support it is peroxidase activity that is being reported by ABTS. Additionally, the $\mathrm{E} 277 \mathrm{Q}$ enzyme variant was unable to generate the bis-Fe(IV) species when analyzed by NIR spectroscopy (Supplementary Fig. 3), a finding also reported for the E113Q variant of $\mathrm{MauG}^{31}$.

Structure of BthA reveals key differences from both bCCPs and MauG. The structure of BthA was solved using X-ray crystallography to 1.54-Å resolution (Supplementary Table 3), revealing the same overall fold found in other structurally characterized bCCPs and MauG (Supplementary Fig. 4). The Ca rmsd of BthA with MauG (PDB 3L4M, 34\% sequence identity) ${ }^{7}$ and Ne bCCP (PDB 1IQC, 25\% sequence identity) $)^{4}$ are $0.974 \AA$ and $3.775 \AA$, respectively. The structural data further show that the $5 \mathrm{c}$ heme pocket is conserved with both bCCPs and MauG, including the presence of the acid-base catalyst, Glu277, which was mentioned above (Supplementary Fig. 5). The $6 \mathrm{c}$ heme pocket in BthA is more similar to MauG, showing Tyr-His ligation by Tyr463 and His 371 (Fig. 4a and Supplementary Fig. 5). Mutation of Tyr463 to histidine $(\mathrm{Y} 463 \mathrm{H})$ results in the inability of the enzyme to generate the NIR feature when treated with $\mathrm{H}_{2} \mathrm{O}_{2}$ (Supplementary Fig. 3), a finding reported for the $\mathrm{Y} 294 \mathrm{H}$ mutation of $\mathrm{MauG}^{32}$. Yet, $\mathrm{Y} 463 \mathrm{H}$ maintained $\mathrm{H}_{2} \mathrm{O}_{2}$ reduction when in the presence of an external electron donor with kinetic values comparable to Wt (Supplementary Table 2).

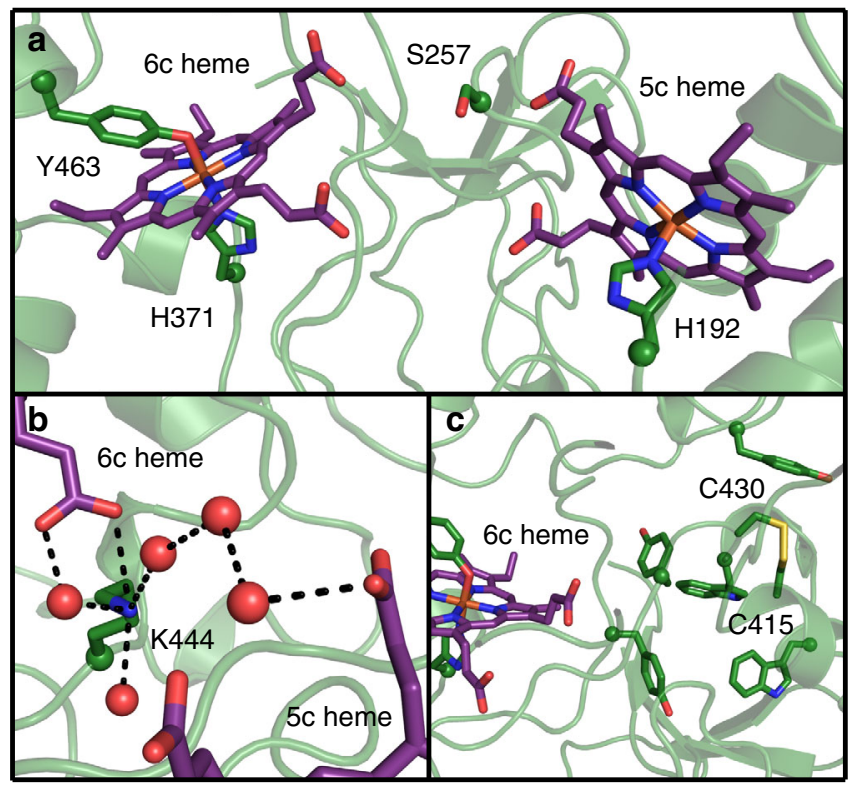

Fig. 4 Structure of BthA. a Ser257 is positioned between the $6 \mathrm{c}$ heme, coordinated by His 371 and Tyr463, and the 5c peroxidatic heme, coordinated by His 192. b Lys 444 mediates a water network between the propionate groups of the $5 \mathrm{c}$ and $6 \mathrm{c}$ hemes. $\mathrm{c} \mathrm{An}$ intramolecular disulfide between Cys 415 and Cys 430 is located near the $6 \mathrm{c}$ heme and is surrounded by tyrosine and tryptophan residues. Peptide and heme carbon shown in green and purple, respectively. Oxygen, nitrogen, sulfur, and iron shown in red, blue, yellow, and orange, respectively. Peptide backbone shown in cartoon representation and sidechains and hemes shown in stick representation. Side-chain $\alpha$-carbon and water shown as spheres 
Both MauG and previously characterized bCCPs contain a tryptophan residue positioned between both hemes $7,20,29,33-35$. Structural and kinetic studies of MauG have shown this interheme tryptophan residue to be necessary for bis-Fe(IV) stabilization and TTQ biosynthesis. In place of this interheme tryptophan residue, BthA has a serine residue, Ser257 (Fig. 4a and Supplementary Fig. 6). We addressed whether this serine residue plays a role in bis-Fe(IV) species formation through mutational analysis, creating S257A to eliminate any possible involvement in ET, and S257W to mimic the conserved tryptophan. Importantly, both S257A and S257W variants generated the bis-Fe(IV) species based on detection of the $960 \mathrm{~nm}$ feature in NIR and yielded nearly identical kinetic values for peroxide turnover (Supplementary Table 2 and Supplementary Fig. 3). A final trait common to MauG and bCCPs is a catalytically essential $\mathrm{Ca}^{2+}$ ion located between the propionate moieties of the two hemes. This ligand maintains an ordered solvent network between the two heme cofactors, and is proposed to regulate the reduction potential and coordination chemistry of the two hemes during catalysis ${ }^{36-38}$. The $\mathrm{Ca}^{2+}$ ion in MauG is tightly bound through coordination to four water molecules, the main-chain carbonyl oxygen atoms of Thr275 and Pro277, and the oxygen atom of the Asn66 sidechain. Additionally, the propionate A carboxylate oxygen of heme $6 \mathrm{c}$ is hydrogen bonded to two of the waters that provide $\mathrm{Ca}^{2+}$ ligands. The arrangement of these key residues and heme propionate group is identical for both bCCPs and MauG. In place of a calcium ligand, BthA contains a lysine residue, Lys444, which mediates a similar solvent network between the heme cofactors (Fig. $4 \mathrm{~b}$ and Supplementary Fig. 7). Notably, the crystal structure revealed the presence of a unique surface-exposed disulfide bond between residues Cys415 and Cys430 (Fig. 4c). Sequence alignment of the Burkholderia orthologs shows the presence of these two cysteine residues at the C-terminal end of the protein sequence is conserved across all Class A proteins. Currently, the function of this disulfide is unknown, but it also sets BthA apart from canonical bCCPs.

\section{Discussion}

Generation of a SSN for the bacterial peroxidase superfamily sets the foundation for discovery of complex peroxide induced chemistry of heme containing proteins. The network shows conservation of canonical bCCPs within the main cluster, representing bCCPs that require reductive activation as well as those reported as constitutively active. The nearest protein neighbors to the biochemically characterized MauG enzyme from $P$. denitrificans appear to be highly conserved in Gram-negative soil bacteria with genomic context suggesting an entirely different area of peroxidatic chemistry. The genomic context of the uncharacterized proteins in the network suggest changes in protein function that extend beyond the traditional role in $\mathrm{H}_{2} \mathrm{O}_{2}$ detoxification, or in the case of MauG, TTQ formation.

BthA is the first example of a diheme enzyme carrying out chemistry reminiscent of both canonical bCCPs and MauG. The electrochemical similarities between BthA and MauG suggest similar propensity for redox chemistry: the voltammetric data collected by direct electrochemistry revealed that both hemes must be similar in potential (where formal potentials arrive from fits to two one-electron redox couples of values of -65 and $-121 \mathrm{mV}$ ), like the two redox transitions observed for MauG. These redox traits may be required to allow for the two electron, metal-centered chemistry associated with the production of the bis-Fe(IV) intermediate. However, the BthA redox potentials are higher than the transitions reported by MauG by $30-55$ $\mathrm{mV}$. The small difference between the individual $5 \mathrm{c}$ and $6 \mathrm{c}$ heme reduction potentials appears to correlate with the observed generation of the bis-Fe(IV) species. However, the slower decay rate of that spectroscopically observed species in BthA may be linked to the difference in the BthA redox potentials in comparison with MauG.

Crystallographic data on BthA shows MauG-like Tyr-His ligation of heme $6 c$ and a similar environment around heme $5 c$ as has been observed in both bCCPs and MauG. Mutational analysis of both the $6 \mathrm{c}$ heme site and the $5 \mathrm{c}$ heme site provided insight into the dual peroxide reactivity of BthA. Y463H and Y463M variants were incapable of generating a feature at $960 \mathrm{~nm}$ when reacted with $\mathrm{H}_{2} \mathrm{O}_{2}$, but surprisingly peroxidase turnover was unchanged in the ABTS-linked assay (Supplementary Table 2). The presence of a Tyr-His ligation to $6 c$ heme site in BthA appears essential for directing BthA toward bis-Fe(IV) formation, but not does not appear to impact the ET mechanism required for $\mathrm{H}_{2} \mathrm{O}_{2}$ reduction (i.e., peroxidase activity with an artificial electron donor). However, E277Q, at 5c heme site, impairs both the bis-Fe(IV) formation and peroxidase activity. The position of this glutamate residue (Glu277) in the distal pocket of the $5 c$ active site of BthA is highly conserved alongside a glutamine residue (Gln267) and proline residue (Pro271), which are analogous to Gln103 and Pro107 in MauG and have been shown to greatly impact heme conformation and reactivity of $\mathrm{MauG}^{39,40}$ Conservation of these three distal pocket residues across bCCPs, MauG and BthA indicates variation of this site is detrimental to the initial peroxide-binding step of either bis-Fe(IV) formation or $\mathrm{H}_{2} \mathrm{O}_{2}$ reduction.

Despite the similarity in protein fold, when comparing BthA and $\mathrm{MauG}$, the lack of a tryptophan residue midway between heme $5 \mathrm{c}$ and $6 \mathrm{c}$ in BthA is a remarkable finding as this feature has been suggested to be critical for both bis-Fe(IV) formation in MauG, and the peroxidase activity of bCCPs ${ }^{30}$. Ser257 replaces the highly conserved Trp93 of MauG, and the replacement of an aromatic side-chain with an aliphatic one would suggest impeded electron transfer. Using the computational tool of HARLEM (see Methods), we examined the potential ET pathways between the $6 c$ and $5 c$ hemes, considering the electron transfer as either a set of two steps that relay through the position equivalent to Ser257 (Trp93 in MauG) or as a single longer step from $6 \mathrm{c}$ heme to $5 \mathrm{c}$ heme, as has been conducted previously for $\mathrm{MauG}^{27}$. Through this approach it is clear that replacement of tryptophan with serine impedes ET, as a direct path for a redox relay to occur is compromised. When considering ET as a series of two steps, the calculated electronic coupling between the $6 \mathrm{c}$ heme and Ser257 is approximately tenfold lower than the analogous step from heme $6 \mathrm{c}$ to Trp93 in MauG $\left(H_{\mathrm{AB}}\right.$ is $3.5 \times 10^{-3}$ for BthA, and $3.7 \times 10^{-2}$ for $\left.\mathrm{MauG}\right)$. In this model, the second step also has a reduced $H_{\mathrm{AB}}$ term, though only by a factor of two $\left(1.4 \times 10^{-2}\right.$ for BthA, compared to $3.8 \times 10^{-2}$ in $\mathrm{MauG}$ ). As $k_{\text {et }}$ is proportional to $\left(H_{\mathrm{AB}}\right)^{2}$, the loss of electronic coupling by a factor of 10 in the first step should greatly impact overall ET rates.

And, when considering the single-step process, HARLEM predicts that direct ET from $6 \mathrm{c}$ to $5 \mathrm{c}$ hemes in BthA occurs through a circuitous route (Supplementary Fig. 8), where the heme proprionate of the $6 \mathrm{c}$ heme serves as pathway for communication to the $5 c$ heme via the backbone residues of His449 and Arg244. This predicted single-step process aligns well with our results for the Ser257 variants, where we still observe a NIR feature upon addition of $\mathrm{H}_{2} \mathrm{O}_{2}$, (Supplementary Fig. 3) and similar steady-state kinetic rates compared to $\mathrm{Wt}$ (Supplementary Table 2). While significant work remains to assess the differences in ET traits between BthA and MauG and how electronic communication may be necessary for bis-Fe(IV) formation, we note that ET between the hemes, therefore, must occur. We then conclude BthA likely reduces $\mathrm{H}_{2} \mathrm{O}_{2}$ with a mechanism distinct from canonical bCCPs. 
The lack of a $\mathrm{Ca}^{2+}$-binding site in the structure of $\mathrm{BthA}$ is also notable in that $\mathrm{Ca}^{2+}$ is known to be essential for enzymatic activity of bCCPs and $\mathrm{MauG}^{37}$. In MauG, the presence of $\mathrm{Ca}^{2+}$ has been shown to impact the heme conformation and spin state, which ultimately impairs the ET that occurs between the heme sites $^{41}$. However in the BthA structure, the $\varepsilon$-ammonium group of Lys444 occupies the position of the conserved $\mathrm{Ca}^{2+}$ ion, one of the notable differences between BthA and MauG, suggesting that the positively charged Lys side-chain mimics a $\mathrm{Ca}^{2+}$ ion. We conducted mutational analysis of Lys444, surveying the affect of a buried charged residue on protein stability and $\mathrm{H}_{2} \mathrm{O}_{2}$ reactivity, and confirming our hypothesis that the positive charge supplied from the $\varepsilon$-ammonium group of Lys444 is essential, effectively replacing the role of $\mathrm{Ca}^{2+}$ observed in other bCCP family members. We produced a K444R variant, which maintains a positive charge at the same position, and showed this variant behaved like Wt and was capable of both $\mathrm{H}_{2} \mathrm{O}_{2}$ reduction (specific activity of $5.3 \mu \mathrm{mol} \mathrm{H}_{2} \mathrm{O}_{2} \mathrm{~min}^{-1} \mathrm{mg}^{-1}$ compared to Wt with $6.1 \mu \mathrm{mol} \mathrm{H}_{2} \mathrm{O}_{2} \mathrm{~min}^{-1} \mathrm{mg}^{-1}$ ) and the production of the NIR feature synonymous with the bis- $\mathrm{Fe}(\mathrm{IV})$ state (Supplementary Fig. 3). However, alteration of the positive charge at this position was deleterious: mutating Lys444 to glutamine (charge removal) or glutamate (charge reversal) resulted in insoluble protein in both cases, suggesting Lys444 is critical for stability if not chemistry.

The $\mathrm{Ca}^{2+}$ ion in MauG is tightly bound through coordination to the main-chain carbonyl oxygen atoms of Thr275 and Pro277, the oxygen atom of the Asn66 side-chain, and four additional water molecules, an arrangement also highly conserved in bCCPs. For BthA, the presence of the Lys444 is in the position of the conserved threonine residue and Ser446 is in place of the highly conserved proline residue. Both seem equally effective in generating a network of water molecules between the two hemes, but any regulation that is based on $\mathrm{Ca}^{2+}$ ion concentrations would be lost in BthA. However, two C-terminal cysteines, which form a disulfide in the BthA structure and are widely conserved in the primary sequence of proteins present in the Burkholderia cluster of the SSN, present a feature of the bCCP family of possible regulatory significance. Disulfide formation can be structural, regulatory or catalytic, and is not a common feature of the bacterial peroxidase family, and thus represents an interesting opportunity for further study.

While the function of BthA has not yet been discovered, the conservation of BthA and the traits of its expression provide hints as to potential biochemical roles. As Burkholderia strains lack conserved mau operons, it is clear that BthA cannot act as a true MauG. It is always found in proximity to a conserved putative purple-acid phosphatase ( $p h o s A$ ), which is predicted to be localized to the outward face of the outer-membrane, and it is conceivable that BthA carries out potential oxidation chemistry on this putative protein partner. Transcriptional analysis by quantitative PCR (qPCR) showed an overexpression of both $b t h A$ and phosA ( +5 and +14 , respectively) during anaerobic growth of B. thailandensis as compared to aerobic growth. This preliminary result helps support our hypothesis that the function of BthA extends beyond a traditional role in $\mathrm{H}_{2} \mathrm{O}_{2}$ scavenging, where $\mathrm{H}_{2} \mathrm{O}_{2}$ is typically produced internally as a misfire of respiration under aerobic conditions.

Generation of a bacterial peroxidase SSN allowed us to biochemically map where canonical bCCPs and MauG lie within the peroxidase superfamily, and identify an area of uncharacterized peroxide chemistry present in Burkholderia. By use of bioinformatics and biophysical methods, we have identified a previously unreported diheme enzyme that stands out from the traditional bCCPs and MauG enzymes. Probing the ET network of BthA, we have identified some of the residues essential for the directing the enzyme toward bis-Fe(IV) formation, while maintaining the ability to efficiently reduce $\mathrm{H}_{2} \mathrm{O}_{2}$.

\section{Methods}

Sequence similarity network for CCP_MauG domain proteins. A sequence similarity network was generated using the sequence similarity tool available through EFI. The sequences input for computation are represented with Pfam identifier for CCP_MauG family members, code PF03150. The network was generated using the InterPro 58 and UniProt 2016_06 databases, and consisted of 6600 sequences with the PF03150 identifier. Final network displayed is representative of an alignment score of 30 and $E$-value cutoff of $10^{-60}$, with each node representing sequences with $80 \%$ sequence identity. Cytoscape ${ }^{19}$ was used for the graphical representation of the network.

Molecular biology. bthA from Burkholderia thailandensis (gene ID: BTH_II1092) was synthesized and codon optimized (Supplementary Table 4) for expression in E. coli (GeneWiz). BTH_II1092 was sub-cloned into pETSN expression vector with a cleavable OmpA periplasmic leader sequence and T7 promoter ${ }^{29}$. Cloning was performed routinely using restriction enzymes XhoI and EcoRI, purchased from New England Biolabs (NEB), plasmid mini-prep kits (Qiagen) and T4 DNA ligase (NEB). To avoid potential complications from export to the periplasm and maturation of cytochromes $c$, a truncated BthA sequence, starting at S69 on the $\mathrm{N}$-terminal end was generated. The plasmid (pKR2) contains an N-terminal Strep tag and C-terminal 6xHis tag. The pKR2 vector was further modified to remove the N-terminal Strep tag, and to insert a stop codon and delete the 6xHis tag, generating pKR4, an untagged version of BthA (Supplementary Table 4). Deletion of the N-terminal Strep tag and insertion of the C-terminal stop codon was achieved with QuikChange Site-Directed Mutagenesis Lightning kit (Agilent). Mutations E277Q, Y463H, S257A, S257W, K444R, K444Q, and K444E were generated using QuikChange Site-Directed Mutagenesis Lightning kit (Agilent). E.coli strains were cultured in $2 \mathrm{x}$ YT (Fisher BioReagents) liquid media or $2 \mathrm{x}$ YT agar at $37^{\circ} \mathrm{C}$. Sequences of oligonucleotides used for generation of plasmids and mutants are listed in Supplementary Table 5.

Expression and purification of BthA. BthA was co-transformed with a plasmid containing cytochrome $c$ maturation cassette ${ }^{42}$, into E. coli BL21(DE3) competent cells. Starter cultures of $5 \mathrm{~mL}$ were grown overnight at $37^{\circ} \mathrm{C}$ in $2 \mathrm{x} \mathrm{YT}$, supplemented with $100 \mu \mathrm{g} \mathrm{mL} L^{-1}$ ampicillin and $35 \mu \mathrm{g} \mathrm{mL}^{-1}$ chloramphenicol. The culture was harvested and resuspended in fresh media before inoculating $1 \mathrm{~L}$ flask of $2 \mathrm{x}$ YT for bulk expression at $37^{\circ} \mathrm{C}$ with $220 \mathrm{rpm}$ until $\mathrm{OD}_{600}=0.8$ was reached. Cells were cooled and induced with $100 \mu \mathrm{M} \beta$-d-thiogalactopyranoside (IPTG, Bio-rad) for $17 \mathrm{~h}$ at room temperature with shaking at $150 \mathrm{rpm}$.

Cells were harvested, resuspended in lysis buffer (50 mM HEPES, $300 \mathrm{mM}$ $\mathrm{NaCl}, 5 \%$ glycerol, pH 7.8, $1 \mathrm{mM}$ phenylmethanesulfonyl (PMSF)), and were lysed by sonication. Clarified lysate was obtained after centrifugation at $18,000 \times g$ for $35 \mathrm{~min}$ Supernatant was diluted with Buffer A (50 mM HEPES, $50 \mathrm{mM} \mathrm{NaCl}$, $\mathrm{pH} 7.8$ ) and loaded onto SP-Sepharose cation exchange resin (GE Healthcare). Resin was washed with Buffer A, and eluted with 50\% of Buffer B (50 mM HEPES, $500 \mathrm{mM} \mathrm{NaCl}, \mathrm{pH}$ 7.8). Protein eluted from SP-sepharose was pooled and concentrated to $1 \mathrm{~mL}$ before loading onto S-200 size exclusion column (GE Healthcare) at $4{ }^{\circ} \mathrm{C}$. Purity of BthA containing fractions was assessed by $12.5 \%$ sodium dodecyl sulfate polyacrylamide gel electrophoresis. Pure fractions were concentrated in Amicon 30K MWCO and exchanged into storage buffer $(50 \mathrm{mM}$ HEPES, $100 \mathrm{mM} \mathrm{NaCl}, 10 \%$ glycerol, $\mathrm{pH} 7.8$ ) before being stored at $-80^{\circ} \mathrm{C}$. Protein concentration was determined by Bradford assay.

Ultraviolet (UV)-Visible spectroscopic analysis. UV-Visible spectra for the oxidized and reduced state of BthA were obtained on a Cary50 spectrophotometer (Agilent) using $1 \mathrm{~mL}$ quartz cuvettes (Starna cells). The as-purified, oxidized state was collected under aerobic conditions at $21.0^{\circ} \mathrm{C}$ in $50 \mathrm{mM}$ HEPES, $50 \mathrm{mM} \mathrm{NaCl}$, pH 7.8 buffer. For reduction of the heme centers, either sodium dithionite or sodium ascorbate was added to the cuvettes to a final concentration of 1 or $5 \mathrm{mM}$ under anaerobic conditions with the use of gas-tight syringes (Hamilton). CO binding to the reduced form of the protein was achieved by bubbling in $\mathrm{CO}$ for $30 \mathrm{~s}$ before recording the spectrum.

Steady-state kinetic assay. Peroxidase activity was determined using 2,2'-azinobis(3-ethylbenzothiazoline-6-sulfonic acid) (ABTS, Sigma) as the artificial electron donor. Assay was performed aerobically in assay buffer ( $50 \mathrm{mM}$ phosphate, $\mathrm{pH} 6.5)$ at $21.0^{\circ} \mathrm{C}$, using $\mathrm{H}_{2} \mathrm{O}_{2}$ concentrations ranging from 0.3 to $30 \mu \mathrm{M}$. ABTS and $\mathrm{H}_{2} \mathrm{O}_{2}$ were reacted for $10 \mathrm{~s}$ before addition of enzyme, and background reactivity was subtracted from each trace. Enzyme was added to final concentration of $30 \mathrm{nM}$ (determined by Bradford). The increase of absorbance at $420 \mathrm{~nm}$ was monitored with extinction coefficient $\varepsilon_{420}=36 \mathrm{mM}^{-1} \mathrm{~cm}^{-1}$. Data were recorded on a Cary50 spectrophotometer (Agilent).

Potentiometric titration. Redox titrations were performed anaerobically with an optically transparent thin-layer electrochemical (OTTLE) cell. The OTTLE cell 
set-up utilized 3D printing to construct a cuvette spacer that allowed for small sample volume, as described by Brisdendine et al. ${ }^{25}$. The spacer was printed by Stratasys using polyjet amber clear material. The cell consisted of a working electrode $(0.25 \mathrm{mM}$ thick gold foil and $0.5 \mathrm{mM}$ gold wire (Aldrich)), a M-401 $\mathrm{Ag} / \mathrm{AgCl}$ reference electrode (Microelectrodes, Inc.) and a $1 \mathrm{mM}$ thick platinum wire as a counter electrode. The spacer assembled with the three electrodes mentioned above was placed in a $10 \mathrm{mM}$ quartz cuvette (Starna Cells). A Teflon cap was fit to the cuvette top equipped with a port for the reference, counter, and working electrode that allowed for placement of each electrode in their respective compartment before the cap was sealed and the cuvette purged with argon. The three-component electrode system was set-up to a PGSTAT 12 AutoLab (Ecochemie) potentiostat. The UV-Visible spectrum was obtained on a Cary50 spectrophotometer after the system reached equilibration at the given potential. Multicomponent buffer ( $5 \mathrm{mM}$ HEPES, MES, MOPS, CAPS, TAPS, CHES, $100 \mathrm{mM} \mathrm{NaCl}, \mathrm{pH}$ 7.0) was purged sufficiently with argon before use to remove oxygen. Mediators ranged from -80 to $-290 \mathrm{mV}$ vs. SHE and included potassium indigo-trisulfonate, Safranin-T, FMN, and Janus Green B.

Protein film voltammetry. Electrochemistry experiments were performed using a three-component electrode system in an anaerobic chamber (MBraun). Set-up consisted of a saturated calomel electrode (Fisher) and platinum wire counter electrode. Pyrolytic graphite edge (PGE) electrodes were used as the working electrode. To prepare the PGE electrode, the graphite surface was sanded and polished with an aqueous mix of $1 \mu \mathrm{M}$ alumina before sonication in Milli-Q water for $2 \mathrm{~min}$ Polished electrodes were then left to dry before depositing $16 \mu \mathrm{L}$ of multiwalled carbon nanotube (MWCNT) mixture. Mixture consisted of $1 \mathrm{mg}$ MWCNT (sigma) in $1 \mathrm{~mL}$ of dimethylformamide (DMF). Electrodes were left to dry overnight to ensure surface was sufficiently coated and DMF was no longer present. Before depositing protein, electrodes were rinsed excessively with water and left to dry. For preparation of adsorbed protein onto the MWCNT-coated surface, 1-4 $\mu \mathrm{L}$ of $\sim 400 \mu \mathrm{M}$ protein in $50 \mathrm{mM}$ HEPES, $100 \mathrm{mM} \mathrm{NaCl}, 10 \%$ glycerol, $\mathrm{pH} 7.8$ was pipetted onto the electrode and left to stand for $1 \mathrm{~min}$ before excess protein was pipetted off. Cell solution consisted of multicomponent buffer [ $5 \mathrm{mM}$ HEPES, MES, MOPS, CAPS, TAPS and CHES, $100 \mathrm{mM} \mathrm{NaCl}$ ] to allow for collection at varying $\mathrm{pH}$ from 5.0 to 9.5 . Experiments were carried out at $21^{\circ} \mathrm{C}$. Measurements were recorded on a PGSTAT 12 Autolab (Ecochemie) potentiostat. Data were analyzed using QSoas ${ }^{43}$.

Near-infrared spectroscopic analysis. The spectra for diferric and $\mathrm{H}_{2} \mathrm{O}_{2}$ treated $\mathrm{B}$ thA were recorded on a Shimadzu UV-3600 spectrophotometer in a $1 \mathrm{~mL}$ quartz cuvette (Starna Cells). $\mathrm{H}_{2} \mathrm{O}_{2}$ concentrations used for near-stoichiometric additions to BthA were determined using the extinction coefficient $\varepsilon_{240 \mathrm{~nm}}=43.6 \mathrm{M}^{-1} \mathrm{~cm}^{-1}$. For $\mathrm{Y} 463 \mathrm{H}, \mathrm{E} 277 \mathrm{Q}, \mathrm{S} 257 \mathrm{~W}, \mathrm{~S} 257 \mathrm{~A}$, and $\mathrm{K} 444 \mathrm{R}$ variants, $\mathrm{H}_{2} \mathrm{O}_{2}$ was added to either one or ten equivalents. Spectra were obtained aerobically at room temperature in $50 \mathrm{mM}$ HEPES, pH 7.8 buffer. For S257A, S257W, and K444R spectra were recorded in $50 \mathrm{mM}$ HEPES, $100 \mathrm{mM} \mathrm{NaCl}, 10 \%$ glycerol, $\mathrm{pH} 7.8$.

EPR and Mössbauer spectroscopy. Incorporation of ${ }^{57} \mathrm{Fe}$ was achieved through expression of BthA in minimal media. Spizizen's minimal media $(\mathrm{MM})^{44}$ was prepared for expression of ${ }^{57} \mathrm{Fe}-\mathrm{BthA}$. Media consisted of $15 \mathrm{mM}\left(\mathrm{NH}_{4}\right)_{2} \mathrm{SO}_{4}$, $80 \mathrm{mM} \mathrm{K}_{2} \mathrm{HPO}_{4}, 44 \mathrm{mM} \mathrm{KH}_{2} \mathrm{PO}_{4}$, and $4 \mathrm{mM}$ sodium citrate. For each $1 \mathrm{~L}$ of autoclaved MM, glucose was added to a final concentration of $0.4 \%$ and $10 \mathrm{~mL}$ of trace metal solution consisting of $0.6 \% \mathrm{MgCl}_{2}, 0.05 \% \mathrm{CaCl}_{2}, 0.006 \% \mathrm{MnCl}_{2}$, $0.02 \% \mathrm{ZnCl}_{2}, 0.003 \% \mathrm{CuCl}_{2}, 0.003 \% \mathrm{CoCl}_{2}$, and $0.005 \% \mathrm{Na}_{2} \mathrm{MoO}_{4}$ was added aseptically. The quantity of background ${ }^{56} \mathrm{Fe}$ was determined using ferrozine iron assay ${ }^{45}$ for each flask of media before inoculation. Background ${ }^{56} \mathrm{Fe}$ was determined to be $0.02 \mathrm{ng} \mathrm{L}^{-1} .{ }^{57} \mathrm{Fe}$ metal (Cambridge Isotopes) was added to a final concentration of $2 \mathrm{mg} \mathrm{L}^{-1}$. Expression of ${ }^{57} \mathrm{Fe}-\mathrm{BthA}$ was carried out as described above with the final ${ }^{57} \mathrm{Fe}-\mathrm{BthA}$ sample prepared in the same final sample buffer (vide infra). X-band EPR spectra and Mössbauer data were recorded as reported recently ${ }^{46}$. Briefly, EPR spectra were recorded on a Bruker Elexsys spectrometer equipped with an Oxford ESR 910 cryostat and a Bruker bimodal cavity using a modulation amplitude and frequency of $1 \mathrm{mT}$ and $100 \mathrm{kHz}$ for all spectra. The microwave frequency was calibrated with a frequency counter and the magnetic field was calibrated with a calibrated Hall probe. The temperature was calibrated with a carbon glass resistor (Lakeshore CGR-1-1000) mounted inside an EPR tube. EPR signals were quantified relative to a $1 \mathrm{mM} \mathrm{Cu}(\mathrm{II})$ EDTA standard in $10 \%$ glycerol. The $\mathrm{Cu}$ (II) concentration was quantified by inductively coupled plasma mass spectrometry. Concentrations of spins were determined with the software SpinCount developed by M.P. Hendrich ${ }^{47}$. The software diagonalizes the spin Hamiltonian,

$$
H=\beta_{\mathrm{e}} B \cdot g_{\mathrm{e}} \cdot S+S \cdot D \cdot S,
$$

where all parameters have their usual definitions. The quantitative simulations are least-squares fits of the experimental spectra that accounts for all instrumental intensity factors, which allows the simulated spectra to be assigned a precise sample concentration

${ }^{57} \mathrm{Fe}$ Mössbauer spectra were recorded with two spectrometers using Janis Research dewars. All isomer shifts are reported relative to Fe metal at $298 \mathrm{~K}$. The simulations of Mössbauer spectra were also fit using SpinCount, employing the spin Hamiltonian: ${ }^{47}$

$$
\begin{aligned}
H= & \beta_{\mathrm{e}} B \cdot g_{\mathrm{e}} \cdot S+D\left[S z^{2}-S^{2}+E / D\left(S_{\mathrm{x}}^{2}+S_{\mathrm{y}}^{2}\right)+S \cdot A \cdot I-g_{\mathrm{n}} \beta_{\mathrm{n}} B \cdot I\right. \\
& +\left[3 I_{\mathrm{z}}^{2}-I^{2}+\eta\left[I_{\mathrm{x}}^{2}-I_{\mathrm{y}}^{2}\right]\right]
\end{aligned}
$$

Crystallization of BthA. Initial crystallization screening was performed using the JCSG + suite (Qiagen), and all optimization and cryoprotectant solutions were prepared using chemicals purchased from Hampton Research. Crystal conditions for BthA were identified through sparse matrix screening at $21^{\circ} \mathrm{C}$ using a Crystal Phoenix (Art Robbins Instruments). Initials crystals formed from $150 \mathrm{~nL}$ of BthA (12 $\mathrm{mg} \mathrm{mL}^{-1}$ in $50 \mathrm{mM}$ HEPES, $\mathrm{pH} 7.8,100 \mathrm{mM} \mathrm{NaCl}, 5 \%$ (v/v) glycerol) that was added to $150 \mathrm{~nL}$ of precipitant solution $(200 \mathrm{mM}$ ammonium nitrate, $20 \%(\mathrm{w} / \mathrm{v})$ PEG 3350) and equilibrated against a reservoir of $70 \mu \mathrm{L}$ precipitant solution using the sitting drop method. After 3 weeks, crystals grew out of a heavy precipitate to form red rod-like crystals, which were used to generate a microseed stock in precipitant solution for further optimization.

Through a combination of microseeding and precipitant solution optimization, larger red rod-like crystals were grown by adding $1 \mu \mathrm{L} \mathrm{BthA}\left(12 \mathrm{mg} \mathrm{mL}^{-1}\right.$ in $50 \mathrm{mM}$ HEPES, pH 7.8, $100 \mathrm{mM}$ sodium chloride, $5 \%(\mathrm{v} / \mathrm{v})$ glycerol) to $0.8 \mu \mathrm{L}$ precipitant solution (300 mM ammonium nitrate, 22\% (w/v) PEG 3350) and $0.2 \mu \mathrm{L}$ microseed stock and equilibrating against $500 \mu \mathrm{L}$ precipitant solution using the sitting drop method. After 3 weeks, crystals grew out of a heavy precipitate. Crystals were harvested and cryoprotected in a solution of $200 \mathrm{mM}$ ammonium nitrate, 25\% PEG 3350, and 10\% glycerol for several minutes and flash-frozen in liquid nitrogen.

Data collection and processing. A native data set of $\mathrm{Wt} \mathrm{BthA}$ was collected at a wavelength of $0.9791 \AA$ and temperature of $100 \mathrm{~K}$ on a Pilatus $6 \mathrm{M}$ pixel detector on beamline 24-ID-C at the Advanced Photon Source (Argonne, IL). The data were indexed, integrated, and scaled in spacegroup $\mathrm{P} 22_{1} 2_{1} 2_{1}$ with cell edges $a=51.20 \AA$, $b=84.66 \AA$, and $c=95.80 \AA$ to $1.54-\AA$ resolution using HKL2000 ${ }^{48}$.

Model building and refinement. The structure of BthA was determined to $1.54-\AA$ resolution by molecular replacement in PHASER ${ }^{49}$. The structure of MauG (PDB: $3 \mathrm{~L} 4 \mathrm{M}, 34 \%$ sequence identity $)^{7}$ was used as a search model after pruning to the last common side-chain heteroatom with phenix.sculptor ${ }^{50}$. A solution with 1 molecule per asymmetric unit was found with LLG and TFZ scores of 201 and 16.9, respectively. The initial model had values of $53.0 \%$ and $53.6 \%$ for the working and free $R$-factors, respectively. Iterative rounds of model building and refinement were performed in $\mathrm{COOT}^{51}$ and phenix.refine ${ }^{52}$, respectively. Heme cofactors were added using a $c$-type heme cif file from the CCP4 database, which was modified to reduce planarity restraints, allowing for heme ruffling ${ }^{53}$, and a parameter file was used to covalently link the two heme cofactors to C188 and C191 as well as C367 and C370. Once the polypeptide chain and heme cofactors were built, waters were added automatically and curated manually. Riding hydrogen atoms were added to the protein model using phenix.ready_set ${ }^{52}$. Later refinements and the final structure were verified using composite omit maps. The final model contains residues P126-Q242 and L247-P543 of 543, two covalently bonded c-type heme cofactors, five nitrate ions, two chloride ions, and one glycerol molecule. The first 59 residues of the construct are not modeled. Analysis of the final model was performed with MolProbity ${ }^{54}$, identifying $96.33 \%$, 3.67\%, and $0 \%$ of residues in the favored, allowed, and disallowed regions of the Ramachandran plot. Rotamer analysis shows $0.61 \%$ rotamer outliers. M368, and E379 are listed as rotamer outliers, but their sidechains show clear electron density. The data collection, processing, and refinement statistics are summarized in Supplementary Table 3. Figures were generated using PyMOL (Schrödinger, LLC). All structural software packages were compiled by SBGrid ${ }^{55}$

Calculations of ET pathways of BthA. To evaluate the electron transfer pathways between BthA heme cofactors, the HARLEM program (http://harlem.chem.cmu. edu/index.php) was used for predicting electron transfer characteristics using PATHWAYS ${ }^{56}$. The distance based approach of Dutton ${ }^{57}$ was used to calculate ET parameters, including the relative values of the electron coupling element $\left(H_{\mathrm{AB}}\right)$, the decay constant $(\beta)$, and packing $(\rho)$. The relevant ET parameters were calculated for both a single-step process from the $6 \mathrm{cheme}$ (Donor) to the $5 \mathrm{c}$ heme (Acceptor) using both Wt BthA and a computationally generated S257W mutant, as well as for MauG and the appropriate W93S mutant, as in Geng et al. ${ }^{27}$. These calculations were then compared to two-step processes where the Ser257 (or Trp93) position was used a formal redox intermediary.

Quantitative real-time PCR. B. thailandensis E264 (Wt Bt) was adapted to anaerobic or aerobic growth for at least 30 generations prior to RNA isolation. Cells were routinely grown at $37^{\circ} \mathrm{C}$ in Luria-Broth. Anaerobic cultures were set-up in sealed Balch tubes with $75 \% \mathrm{~N}_{2}, 20 \% \mathrm{CO}_{2}$, and $5 \% \mathrm{H}_{2}$ at 5 psi over ambient pressure and supplemented with $10 \mathrm{mM}$ sodium nitrate as the electron acceptor. RNA was extracted from triplicate cultures grown to stationary phase. Briefly, cells were harvested and resuspended in $1 \mathrm{~mL}$ RNApro solution (MP Biomedical). Cell 
lysate was generated by sonication using a micro-sonicator for $10 \mathrm{~s}$ (Bronson). Lysate was centrifuged for $5 \mathrm{~min}$ at $13,000 \mathrm{rpm}, 4^{\circ} \mathrm{C}$. Supernatant was transferred to a new tube and incubated at room temperature for $5 \mathrm{~min}$ before addition of $300 \mu \mathrm{L}$ chloroform. Sample was transferred to a $2 \mathrm{~mL}$ pre-spun phase-lock tube (QuantaBio) and incubated for $5 \mathrm{~min}$ before centrifugation at 13,000 rpm. Upper phase was mixed with $500 \mu \mathrm{L}$ of $100 \%$ RNase free ethanol and incubated at $20^{\circ} \mathrm{C}$ for $1 \mathrm{~h}$. RNA was pelleted at $13,000 \mathrm{rpm}, 4^{\circ} \mathrm{C}$ for $15 \mathrm{~min}$ and washed with $500 \mu \mathrm{L}$ of $75 \% \mathrm{EtOH}$ and let to dry before resuspension in $100 \mu \mathrm{L}$ nuclease-free $\mathrm{H}_{2} \mathrm{O}$ (Invitrogen). Partially purified RNA was cleaned up using the RNeasy Mini Kit (Qiagen) following manufacturer's recommendation. RNA concentrations were quantified and placed at $-80^{\circ} \mathrm{C}$ until needed. Complementary DNA (cDNA) was synthesized using TaqManR reverse transcription reagents (Applied Biosystems) following manufacturer's protocol. Quantitative PCR analysis was carried out using 2X SYBR Green ROX PCR master mix (ThermoFisher) in a reaction of $100 \mathrm{ng}$ template cDNA, and $0.3 \mu \mathrm{M}$ reverse and forward primers for each gene were analyzed in a total volume of $20 \mu \mathrm{L}$. Primers used for amplification of $b$ thA (bthAF, bthA-R), phosA (phosA-F, phosA-R), and housekeeping gene gyrB (gyrB-F, $\operatorname{gyr} B-R$ ) are listed in Supplementary Table 5. Control gene gyrB was selected based on previous reports for similar experiments in B. thailandensis ${ }^{58}$. Reactions were carried out on a 7900ht instrument (Applied Biosystems). Relative quantification of gene expression by qPCR was determined by use of the $2^{\Delta \Delta C t} \operatorname{method}^{59}$.

\section{Data availability}

The sequences used in the SSN are from the Uniprot database, with accession codes listed in Supplementary Table 1. Crystallographic data has been deposited with accession code $6 \mathrm{NX} 0 . \mathrm{pdb}$ at the Protein Data Bank. Other data are available from the corresponding author upon reasonable request.

Received: 15 December 2018 Accepted: 15 February 2019

Published online: 07 March 2019

\section{References}

1. Goodhew, C. F., Wilson, I. B., Hunter, D. J. \& Pettigrew, G. W. The cellular location and specificity of bacterial cytochrome c peroxidases. Biochem. J. 271, 707-712 (1990).

2. Welinder, K. G. Superfamily of plant, fungal and bacterial peroxidases. Curr. Opin. Struct. Biol. 2, 388-393 (1992). (92)90230-5.

3. Poulos, T. L. Heme enzyme structure and function. Chem. Rev. 114, 3919-3962 (2014).

4. Shimizu, H. et al. Crystal structure of Nitrosomonas europaea cytochrome c peroxidase and the structural basis for ligand switching in bacterial di-heme peroxidases. Biochemistry 40, 13483-13490 (2001).

5. Wang, Y. et al. MauG, a novel diheme protein required for tryptophan tryptophylquinone biogenesis. Biochemistry 42, 7318-7325 (2003).

6. Li, X., Feng, M., Wang, Y., Tachikawa, H. \& Davidson, V. L. Evidence for redox cooperativity between c-type hemes of MauG which is likely coupled to oxygen activation during tryptophan tryptophylquinone biosynthesis. Biochemistry 45, 821-828 (2006)

7. Jensen, L. M., Sanishvili, R., Davidson, V. L. \& Wilmot, C. M. In crystallo posttranslational modification within a MauG/pre-methylamine dehydrogenase complex. Science 327, 1392-1394 (2010).

8. Arciero, D. M. \& Hooper, A. B. A di-heme cytochrome c peroxidase from Nitrosomonas europaea catalytically active in both the oxidized and halfreduced states. J. Biol. Chem. 269, 11878-11886 (1994).

9. Li, X., Fu, R., Liu, A. \& Davidson, V. L. Kinetic and physical evidence that the diheme enzyme MauG tightly binds to a biosynthetic precursor of methylamine dehydrogenase with incompletely formed tryptophan tryptophylquinone. Biochemistry 47, 2908-2912 (2008).

10. Shin, S., Abu Tarboush, N. \& Davidson, V. L. Long-range electron transfer reactions between hemes of MauG and different forms of tryptophan tryptophylquinone of methylamine dehydrogenase. Biochemistry 49, 5810-5816 (2010).

11. Geng, J., Davis, I., Liu, F. \& Liu, A. Bis-Fe(IV): nature's sniper for long-range oxidation. J. Biol. Inorg. Chem. : JBIC : a Publ. Soc. Biol. Inorg. Chem. 19, 1057-1067 (2014).

12. $\mathrm{Li}, \mathrm{X}$. et al. A catalytic di-heme bis-Fe(IV) intermediate, alternative to an $\mathrm{Fe}(\mathrm{IV})=\mathrm{O}$ porphyrin radical. Proc. Natl Acad. Sci. USA 105, 8597-8600 (2008).

13. Rittle, J. \& Green, M. T. Cytochrome P450 compound I: capture, characterization, and C-H bond activation kinetics. Science 330, 933-937 (2010).

14. Krest, C. M. et al. Reactive intermediates in cytochrome p450 catalysis. J. Biol. Chem. 288, 17074-17081 (2013).

15. Delorme, C. et al. Expression of the mau gene cluster of Paracoccus denitrificans is controlled by MauR and a second transcription regulator. Microbiology 143(Pt 3), 793-801 (1997).
16. Govan, J. R. \& Deretic, V. Microbial pathogenesis in cystic fibrosis: mucoid Pseudomonas aeruginosa and Burkholderia cepacia. Microbiol. Rev. 60, 539-574 (1996).

17. Gerlt, J. A. et al. The Enzyme Function Initiative. Biochemistry 50, 9950-9962 (2011).

18. Gerlt, J. A. et al. Enzyme function initiative-enzyme similarity tool (EFI-EST) A web tool for generating protein sequence similarity networks. Biochim. Biophys. Acta 1854, 1019-1037 (2015).

19. Shannon, P. et al. Cytoscape: a software environment for integrated models of biomolecular interaction networks. Genome Res. 13, 2498-2504 (2003).

20. Fulop, V., Ridout, C. J., Greenwood, C. \& Hajdu, J. Crystal structure of the di-haem cytochrome c peroxidase from Pseudomonas aeruginosa. Structure 3, 1225-1233 (1995).

21. Pulcu, G. S. et al. The diheme cytochrome c peroxidase from Shewanella oneidensis requires reductive activation. Biochemistry 51, 974-985 (2012).

22. Khademian, M. \& Imlay, J. A. Escherichia coli cytochrome c peroxidase is a respiratory oxidase that enables the use of hydrogen peroxide as a terminal electron acceptor. Proc. Natl Acad. Sci. USA 114, E6922-E6931 (2017).

23. Izadi-Pruneyre, $\mathrm{N}$. et al. The heme transfer from the soluble HasA hemophore to its membrane-bound receptor HasR is driven by protein-protein interaction from a high to a lower affinity binding site. J. Biol. Chem. 281, 25541-25550 (2006).

24. Moore, G. R. \& Pettigrew, G. W. Cytochromes c: Evolutionary, Structural and Physicochemical Aspects (Springer-Verlag, Berlin Heidelberg, 1990).

25. Brisendine, J. M., Mutter, A. C., Cerda, J. F. \& Koder, R. L. A threedimensional printed cell for rapid, low-volume spectroelectrochemistry. Anal. Biochem. 439, 1-3 (2013).

26. Debrunner, P. G. in Iron Porphyrins Part III, Vol. IV, eds Lever A. B. P., Gray H. B., 137-234, VCH New York (1989).

27. Geng, J., Dornevil, K., Davidson, V. L. \& Liu, A. Tryptophan-mediated chargeresonance stabilization in the bis-Fe(IV) redox state of MauG. Proc. Natl Acad. Sci. USA 110, 9639-9644 (2013).

28. Yukl, E. T., Williamson, H. R., Higgins, L., Davidson, V. L. \& Wilmot, C. M Oxidative damage in MauG: implications for the control of high-valent iron species and radical propagation pathways. Biochemistry 52, 9447-9455 (2013)

29. Hoffmann, M., Seidel, J. \& Einsle, O. CcpA from Geobacter sulfurreducens is a basic di-heme cytochrome c peroxidase. J. Mol. Biol. 393, 951-965 (2009).

30. Pettigrew, G. W., Echalier, A. \& Pauleta, S. R. Structure and mechanism in the bacterial dihaem cytochrome c peroxidases. J. Inorg. Biochem. 100, 551-567 (2006).

31. Abu Tarboush, N. et al. Carboxyl group of Glu113 is required for stabilization of the diferrous and bis-Fe(IV) states of MauG. Biochemistry 52, 6358-6367 (2013).

32. Abu Tarboush, N. et al. Functional importance of tyrosine 294 and the catalytic selectivity for the bis-Fe(IV) state of MauG revealed by replacement of this axial heme ligand with histidine. Biochemistry 49, 9783-9791 (2010).

33. De Smet, L., Savvides, S. N., Van Horen, E., Pettigrew, G. \& Van Beeumen, J. J Structural and mutagenesis studies on the cytochrome $c$ peroxidase from Rhodobacter capsulatus provide new insights into structure-function relationships of bacterial di-heme peroxidases. J. Biol. Chem. 281, 4371-4379 (2006).

34. Zahn, J. A., Arciero, D. M., Hooper, A. B., Coats, J. R. \& DiSpirito, A. A. Cytochrome c peroxidase from Methylococcus capsulatus Bath. Arch. Microbiol. 168, 362-372 (1997).

35. Schutz, B., Seidel, J., Sturm, G., Einsle, O. \& Gescher, J. Investigation of the electron transport chain to and the catalytic activity of the diheme cytochrome c peroxidase CcpA of Shewanella oneidensis. Appl. Environ. Microbiol. 77, 6172-6180 (2011)

36. Timoteo, C. G. et al. Ca2+ and the bacterial peroxidases: the cytochrome $\mathrm{c}$ peroxidase from Pseudomonas stutzeri. J. Biol. Inorg. Chem. : JBIC : a Publ Soc. Biol. Inorg. Chem. 8, 29-37 (2003).

37. Chen, Y. et al. Role of calcium in metalloenzymes: effects of calcium removal on the axial ligation geometry and magnetic properties of the catalytic diheme center in MauG. Biochemistry 51, 1586-1597 (2012).

38. Shin, S., Feng, M. \& Davidson, V. L. Mutation of Trp(93) of MauG to tyrosine causes loss of bound $\mathrm{Ca}(2+)$ and alters the kinetic mechanism of tryptophan tryptophylquinone cofactor biosynthesis. Biochem. J. 456, 129-137 (2013).

39. Feng, M. et al. Proline 107 is a major determinant in maintaining the structure of the distal pocket and reactivity of the high-spin heme of MauG. Biochemistry 51, 1598-1606 (2012).

40. Shin, S., Yukl, E. T., Sehanobish, E., Wilmot, C. M. \& Davidson, V. L. Sitedirected mutagenesis of Gln103 reveals the influence of this residue on the redox properties and stability of MauG. Biochemistry 53, 1342-1349 (2014).

41. Shin, S. et al. The tightly bound calcium of MauG is required for tryptophan tryptophylquinone cofactor biosynthesis. Biochemistry 50, 144-150 (2011).

42. Thony-Meyer, L., Fischer, F., Kunzler, P., Ritz, D. \& Hennecke, H. Escherichia coli genes required for cytochrome c maturation. J. Bacteriol. 177, 4321-4326 (1995). 
43. Fourmond, V. QSoas: A versatile software for data analysis. Anal. Chem. 88, 5050-5052 (2016).

44. Anagnostopoulos, C. \& Spizizen, J. Requirements for Transformation in Bacillus Subtilis. J. Bacteriol. 81, 741-746 (1961).

45. Carter, P. Spectrophotometric determination of serum iron at the submicrogram level with a new reagent (ferrozine). Anal. Biochem. 40, 450-458 (1971).

46. Sui, X. et al. Structure and spectroscopy of alkene-cleaving dioxygenases containing an atypically coordinated non-heme iron center. Biochemistry 56, 2836-2852 (2017).

47. Golombek, A. P. \& Hendrich, M. P. Quantitative analysis of dinuclear manganese(II) EPR spectra. J. Magn. Reson. 165, 33-48 (2003).

48. Otwinowski, Z. \& Minor, W. Processing of X-ray diffraction data collected in oscillation mode. Methods Enzymol. 276, 307-326 (1997).

49. McCoy, A. J. et al. Phaser crystallographic software. J. Appl. Crystallogr. 40, 658-674 (2007).

50. Bunkoczi, G. \& Read, R. J. Improvement of molecular-replacement models with Sculptor. Acta Crystallogr. D. Biol. Crystallogr. 67, 303-312 (2011).

51. Emsley, P. \& Cowtan, K. Coot: model-building tools for molecular graphics. Acta Crystallogr. D. Biol. Crystallogr. 60, 2126-2132 (2004).

52. Adams, P. D. et al. PHENIX: a comprehensive Python-based system for macromolecular structure solution. Acta Crystallogr. D Biol. Crystallogr. 66, 213-221 (2010).

53. Roberts, S. A. et al. Ligand-induced heme ruffling and bent no geometry in ultra-high-resolution structures of nitrophorin 4. Biochemistry $\mathbf{4 0}$, 11327-11337 (2001).

54. Chen, V. B. et al. MolProbity: all-atom structure validation for macromolecular crystallography. Acta Crystallogr. D. Biol. Crystallogr. 66 12-21 (2010).

55. Morin, A. et al. Collaboration gets the most out of software. eLife 2, e01456 (2013).

56. Balabin, I. A., Hu, X. \& Beratan, D. N. Exploring biological electron transfer pathway dynamics with the Pathways plugin for VMD. J. Comput. Chem. 33, 906-910 (2012).

57. Page, C. C., Moser, C. C., Chen, X. \& Dutton, P. L. Natural engineering principles of electron tunnelling in biological oxidation-reduction. Nature 402, 47-52 (1999).

58. Ferreira, A. S. et al. Distribution of cepacian biosynthesis genes among environmental and clinical Burkholderia strains and role of cepacian exopolysaccharide in resistance to stress conditions. Appl. Environ. Microbiol. 76, 441-450 (2010).

59. Pfaffl, M. W. A new mathematical model for relative quantification in real-time RT-PCR. Nucl. Acids Res. 29, e45 (2001).

\section{Acknowledgements}

K.R. and S.J.E. are supported by the National Institutes of Health/NIGMS (NIH R01GM110390). S.E.C. is supported by NIH T32 GM008334 C.L.D. is a Howard Hughes Medical Investigator. This research is supported in part by NIH R35 GM126982 (C.L.D.). C.L.D. is a Senior Fellow of the Bio-inspired Solar Energy Program, Canadian Institute for Advanced Research (CIFAR). This work is based upon research conducted at the Northeastern Collaborative Access Team beamlines, which are funded by the NIH (P41 GM103403). The Pilatus 6M detector on 24-ID-C beamline is funded by a NIHORIP HEI grant (S10 RR029205). This research used resources of the Advanced Photon Source, a U.S. Department of Energy (DOE) Office of Science User Facility operated for the DOE Office of Science by Argonne National Laboratory under Contract No. DEAC02-06CH11357. Software used in the project was installed and configured by SBGrid.

\section{Author contributions}

K.R. conducted the bioinformatics analysis, identified genes, constructed all plasmids, purified all proteins, and performed and analyzed the optical/NIR spectroscopic data, steady-state kinetics, and electrochemistry experiments. A.C.W. collected and analyzed EPR and Mössbauer data, S.E.C. conducted all crystallography experiments and analysis. M.M.M.L. designed the qPCR experiments, analyzed the data, and authored those sections of the manuscript. M.P.H., C.L.D., and S.J.E. designed the experiments, supervised the research, analyzed the data and wrote the manuscript in collaboration with all other authors.

\section{Additional information}

Supplementary Information accompanies this paper at https://doi.org/10.1038/s41467019-09020-4.

Competing interests: The authors declare no competing interests.

Reprints and permission information is available online at http://npg.nature.com/ reprintsandpermissions/

Journal peer review information: Nature Communications thanks Carrie Wilmot and the other anonymous reviewer(s) for their contribution to the peer review of this work.

Publisher's note: Springer Nature remains neutral with regard to jurisdictional claims in published maps and institutional affiliations.

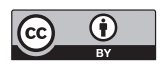

Open Access This article is licensed under a Creative Commons Attribution 4.0 International License, which permits use, sharing, adaptation, distribution and reproduction in any medium or format, as long as you give appropriate credit to the original author(s) and the source, provide a link to the Creative Commons license, and indicate if changes were made. The images or other third party material in this article are included in the article's Creative Commons license, unless indicated otherwise in a credit line to the material. If material is not included in the article's Creative Commons license and your intended use is not permitted by statutory regulation or exceeds the permitted use, you will need to obtain permission directly from the copyright holder. To view a copy of this license, visit http://creativecommons.org/ licenses/by/4.0/.

(C) The Author(s) 2019 